\title{
XXVIII.
}

Aus dem pharmakologischen Institut der Universität Leipzig.

\section{Dynamik des Froschherzventrikels bei nicht tödtlicher Digitalisvergiftung.}

\author{
Yon \\ Walther Straub. \\ (Hiewa lafo! XXl und 10 figuren in Text)
}

Die vorliegende Untersuchung enthält die Analyse der Wirkung mehrerer Digitalisglykoside am Ventrikel des ausgeschnittenen Froschherzens auf Grund der gegenseitigen Beziehungen zwisehen Füllung und Frequenz, Anfangsspannung und Pulsvolum (isotonische Zuckung), sowie fïllung und Druck (isometrischo Zuckung).

Dio Durchführung fusst auf den durch 0 . Frank's ${ }^{1}$ ) Untersuchungen festgestellten Principien der Dynamik des Herzmuskels, auf dic ich zuror noch genauer eingehen zu müssen glaube.

Frank hat die Herzthätigkeit rein mechanisch nach den Principien zerglicdert, die Fick für die 'Thätigkeit des Skelettmuskels aufgestellt hat, und den Beziehungen der mechanischen Homente der Ruhe zu denen der Thätigkeit dic Terminologic der Juskelmechanik angepasst.

lis ergab sich, dass für den Herzmuskel cbenso wie für den Skeletımuskel die Spannung in der Ruhelage massgebend ist für den Grad der 'Thätigkeit in den beiden mechanischen Grenzfällen, der Thätigkcit unter Verkürzung ohne Spannungsveränderung (isotonische Zuckung:) und der unter Spannungsänderung ohne Verkürzung (isometrische Zuckung). Die Beziehung der Verkürzungsgrösse (gemessen am Pulsvolum) zur Grösse der Ruhebelastung (Anfangsspannung, Füllung) für alle möglichen Werthe der letzteren nennt Frank , die Dehnungscurve der isotonisehen Llaxima", und die der Spannung zur Ruhebelastung "die Dehnungscurve der isometrischen Maxima". Die Beziehung der Ruhılänge der einzelnen Nuskelelemente zur jeweiligen Anfangsspannung, die beim Skelettmuskel die "Dehnungscurve des ruhenden Muskels" ausdrückt, lässt sich bei dem rhythmisch thätigen Herzmuskel nicht crlangen; Frank hat an

1) O. Frank, Zur Dynamik des Herzmuskels. 'L. f. Biol. 32. 1895. - Dersclbe, Die Wirlung ron Digitalis (Helleborëin) auf das IJerz. Sitzungsber. d. Cies. C. Morphol. u. Physiol. München. 1897. Il. - Derselbe, Isometrie und Isotonie des Herzmuskels, Z. f. Biol. 41. 1899. 
ihrer Stelle die Beziehung der maximalen diastolischen Füllung zur Anfangsspannung, ausgedrückt durch die „Dehnungscurve der isotonischen Minima", gesetzt.

Alle diese Beziehungen (Dehnungseurven) sind aus Reihenbeobachtungen zu construiren und nicht unmitlelbar aus der registrirten Thätigkeit zu entnehmen.

Die natürliche Thätigkeit des Ventrikels crfolgt als Combination der eben mitgetheilten Beziehungen; sie beginnt isometriseh und endet isotonisch. Der Uebergangspunkt von einer Phase zur anderen ist scharf in der Oeffnung der Aortenklappen gegeben, von dem Moment an lastet aut dem Ventrikel der Blutdruck, gegen flen dann die woitere Zuckung isotoniseh erfolgt. Der maximale Druekwerth der isometrisehen Phase wird also grösser sein als der Blutdruck. Unter Anlehnung an die Terminologie der Muskclmechanik verläuft demnach die natürliche Zuckung des Ventrikels als Unterstützungszuckung, wobei das unterstützende Moment die Aortenklappen sind, dic in der Diastole den Blutdruck von den Ventrikelwänden absperren.

Die gegenseitigen Beziehungen zwischen Druck und Volum bei dieser Art von Zuckungen sind verwickeltere, Frank konnte indess darthun, dass die diese Beziehungen repräsentirende "Dehnungscurve der Maxima der Unterstützungszuckung "zwischen den Dehnungseurven der isotonischen und isometrischen Naxima verlaufen muss.

Für die Beurtheilung der Wirkung eines Agens auf das als Organ functionirende Herz kommt von der Gesammtheit der durch die „Dehnungscurven" bestimmten Möglichkeiten nur ein sehr geringer Antheil in Betracht, der begrenzt ist durch das Maximum an Druck, das in der Diastole das Naximum an Füllung erzeugt, d. i. nur der Anfangstheil der "Dehnungscurve der isotonischen Minima", oder für dic Organfunction dic Druckmaxima der (isotonischen) Vorhofszuckung, also jedenfalls nur einige Millimeter Quecksilber.

Auch aus der Beobachtungsthatsache, dass in der Organfunction des Herzens Ventrikelfüllung und Pulsvolum nahezu identisch sind, lässt sich der Bereich des praktisch Verwirklichten von dem des theoretisch Möglichen trennen. Nach Frank (Die Wirkung von Digitalis, These 3) steigen nämlich die ausgeworfenen Volumina mit wachsenden Drucken, um später wieder abzunehmen. Es wird also nur der Anfangstheil der "Dehnungscurve der isotonischen Maxima" bis höchstens ztum Umkehrpunkt von praktischer Bedeutung sein.

In einer früheren Arbeil über das allgemein physiologische Verhalten des Ventrikelmuskels unter Digitaliswirkung ${ }^{1}$ ) habe ich festgestelit: dass die Digitalisglykoside dic Erregbarkeit und die lirregung der Herzmuskelzellen herabsetzen, also cine negativ bathmotrope und negativ inotrope Wirkung äussern"). lch habe erläutert, wic dic negativ bathmo-

1) W. Straub, Ueber die Wirkung des Antiarin's am ausgeschnittenen, suspendirten Froschherzen. A. ¡. exp. Path. u. Pharm. 45. S. 3̈46.

2) Ich habe in der citirten Abhandlung die constatirte Einwirkung auf zwei Grundvermögen der Herzmuskelzellen im Sinne H. E. Ilering's als zwei unlösbar 
trope Wirkung zu cinem Sinken der Frequenz der Pulse auf die Hälfte fiihren muss, und constatirt, dass man durch geeignete Dosirung diese Halbirung zum bleibenden Zustande machen kann. Die Halbirung kann, wie ferner gezeigt wurde nach den schon von Bowditseh constatirten und neuerdings von F. B. Holmann ${ }^{1}$ ) genauestens analysirten Beziehungen zwischen Pausenlänge und Zuckungshöhe in der rhythmischen Thätigkeit, die gleichzeitig aufgetretene negativ inotrope Wirkung übercompensiren, was bei der Function des IIerzmuskels im Organ eine Vermehrung des Pulsvolum bedeutet.

Es fragt sich nun, welche Bedeutung haben die beobachteten negativen Beemflussungen zweier Grundeigensehaften des Herzmuskels für die Jeistung des Organs, kann speciell mit ihnen cin Effect erzielt werden, der im Sinne der Heilwirkung der Digitalisglykoside als ein positiver aufzufassen ist.

Es erwuchs also die Aufgabe, im ganzen Verlaufe einer durch die Giftmenge bestimmten endlichen Zustandsänderung nach den Frank'schen Prineipien die mechanisehe Analyse der Ventrikelthätigkeit durchzuführen.

Um den Plan in möglichst einfacher Weise durchzuführen, habe ich blos zwei mechanische Momente verfolgt, nämlich einmal die Bezichung der Anfangsspannung zur Ventrikelfüllung (Dehnungseurve der isotonisehen Minima) und dann die der Ventrikellüllung zum Druckmaximum bei isometrischer Thätigkeit, also dem Falle der maximalen Kraftentlaltung (Dehnungscurve der isometrischen Maxima). Die erste Bezichung giebt Aufschluss über den zeitlichen Zustand der Elasticität des pulsirenden Ventrikels (aber nicht des ruhenden Herzmuskels [Erank]), die zweite, die der natürlichen Zuckung in bestimmter Weise coordinirt ist (s. o.), lässt die Kreislaufsünderungen bis zu einem gewissen Grade discutiren. Die Beziehung Druck: Pulsvolum bei isotonischer Thätigkeit habe ich nur für die niedersten Druckwerthe ermittelt, nämlich für jene, die zur Herbeiführung der maximalen diastolischen Füllungen nöthig wird, also cinige Millimeter Quecksilber. Die Ermittlung der ganzen „Dehnungscurve der isotonischen Maxima" hätte cine complicirtere Apparatur verlangt und damit die Zuverlässigkeit der hesultate der lange dauernden Versuche beeinträchtigt.

Für einen Punkt der Vergiftung mit einem Digitalisglykosid nämlich dic Frequenzhalbirung durch Holleborëin -- ist die Untersuchung von 0 . Frank ${ }^{2}$ ) schon durchgeführt. Ich werde später eingehend auf diese Untersuchung zurückkommen.

Zur Durchführung des Programms habe ich mir eine speciclie Vorsuchsanordnung ausgearbeitet, die von der Frank'sehen äusserlich etwas abweicht. Sie soll zunächst ausführlicher besprochen werden, weil ich

miteinander verkuppelte Wirkungen aufgelasst. Inzwischen bin ich von der Stichhaltigkeit dieses toxikologischen Argumentes abgekommen und erkenne die Einwände von H. Meyer und N. H. Alcock (Engelmann's Arehiv. 1903. 235) an.

1) F. B. Hofmann, Pliuger's Archiv. 1901. Bd. 84. S. 140.

2) O. Frank, Sitzungsber. d. Ges. f. Morphol. 1. Physiol. München. 1897. S. 26 d. Sep.-Abdr. 
sic für gecignet erprobt habe, zur Prifung des dynanischen Verhaltens des Herzens unter pharmakologischen Eingriffen.

\section{Versuchsanordumug.}

Es kam darauf an, den Verlauf der Vergiftung durch Dosirung zu beherrschen und einen duroh cin bestimmtes Sympton grekennzeichneten Punkt mit Sicherheit zu erreichen. Mls diesen Punkt wählte ich den Lintritt der Halbirung. Dabei leitete mich die chemische Torstellung, dass mit dem Eimbringen des Giftes in den Organismus oder das System, in dem das isolirte Organ arbeitet, cin Wandern des Giftes in das Herz vor sich geht, wobei schliesslich ein Gleichgewiehtszustand erreicht wird, bei dem alles oder fast alles Gift im Herzen enthalten ist, so dass also die Tiefe der Vergiftung von der absoluten Menge des Giftes bedingt ist. Demgemäss hat dic Concentration blos dic Bedeutung eines Gesehwindigkeitsfactors. Zur Lrrciehung des oben angedeuteten Zweckes ergiebt sich die Forderung, die gewählte Dosis in nieht zu starker Verdünnung wirken zu lassen, $m$. a. $W$. das im System befindliche Flüssigkeitsquantum gering zu halten. Ich bin schlicsslich mit 5 cem Ringer'scher lösung ausgekommen, ein Wertl, der der absoluten Blutmenge der verwandten Frösche ziemlich nahe kommen dürfte.

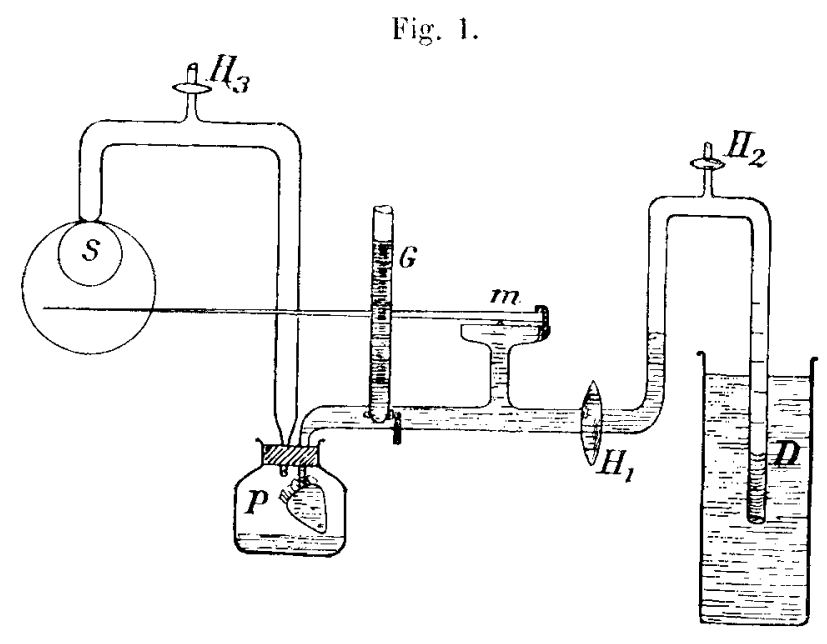

Der von mir verwandte, in Fig. 1 sehematisch dargestellte Apparat ist in der Idec genau dasselbe wie die Erank'sche Versuchsanordnung (O. Frank: Dic Wirkung von Digitalis ete. S. 15 d. Sep.-Abdr.). Der Ventrikel arbeitet dauernd mit dem im Röhrensystem enthaltenen Quantum Flussigkeit. Das Her\% ist in einem luft-Plethysmographen; die Anfangsspannungen werden eryeugt und varirt dureh Heben und Senken des Flïssigkeitsgefässes D bei geschlossenem Ilahn H, und offenem Hahn $\mathrm{H}_{1}$; wird durch Schluss des Hahms $\mathrm{H}_{1}$ in Diastole des Ventrikels die Flüssigkeit gegen D hin abgesperri, so erfolgt die närhste Zuckung isometrisch; der Druek wird durch das Gummimanometer $M$ angezeigt. Die Aufnahme der Dehnungseme der isometrisehen Naxima erfolgt dann in 
der Weise, dass man bei offenem Hahn $H_{1}$ das Gefäss $D_{\text {D }}$ soweit senkt, dass eben keine Bewegung mehr in her Füllfüssigkeit orfolgt, dieser Stand wird als Anfangsspannung O notirt. Dann wird D um $1 \mathrm{~cm}$ gehoben, in Diastole Hahn 1 gespert und darauf die isometrisehe Zuckung bei Anfangsspannung $1 \mathrm{~cm} \mathrm{H}_{2} \mathrm{O}$ registrirt u. s. w. bis zu ciner Anfangsspannung, die keinen sichtlichen Zuwachs an Pulsvolum mehr zur Folge hat. Die Volumsehwankung des Ventrikels misst die Seifonblase $\mathrm{S}^{1}$ ), die mit dem Plethysmographeninneren communicirt. Steht Hahn 1 offen, so registrirt die Seifenblase die zu der betreffenden Anfangspannung gehörende isotonische Zuckung, successives Heben des Gefässes I) ergiebt dann den Anfangstheil dor Dehnungsurve der isotonischen Maxima.

Die Bewegung der Seifenblase orientirt, wie bemerkt, über das Verhältniss des Pulsvolums zur Fïlllung und Anfangsspannung, d. h. den Verlauf der Dehnungseure ler isotonisehen Iaxima. Aus oben crörterten Gründen habe ieh mit einer geringen Breite von $\Lambda$ nfangsspannungen operirt, nämlich jener, die zur maximalen Füllung ausreichen. Da andererseits in meinen Versuchen diese Anfangsspannungen gleichzeitig die Belastungen bei der isotonischen Thätigkcit sind, könnte ich iiber den Verlauf der Dehnungseurve der isotonischen Maxima nur für ihren kleinsten, oben definirten Theil aussagen. Es hat sich herausgestellt, dass bei isotoniseher Thätigkeit unter den kleinen Belastungen Pulsyolum und Herzfüllung auch während der Digitaliswirkung identisch sind, die Dehnungscurve der isotonischen Maxima in ihrem Anfangstheil also ungeändert verläuft. Ich nehme dieses practisch bedeutungslose Resultat vorweg und lasse im Folgenden die Dehnungseurve der isotonischen llaxima ausser Betracht.

In dem Rohr $G$ ist die Giftlösung enthalten, die im gewünscliten Moment nach Herstellung einer negativen Anfangsspannung zugclassen wird. (Nach Zugabe der Giftlösung muss natürlich eine neue Anfangsspannung 0 ermittelt werden.)

Die Verzeichnung der Manomoterausschläge und der Pulsation der Seifenblase crfolgte auf photographischem Wege, also ohne Arbeitsverlust durch Reibung, und zwar in vergrössertem Massstabe dureh Zwisehenschaltung eines photographischen Objectirs zwischen Object und Rigistrirapparat. Die Aiehung des Manometers geschah mit dem Quecksilbermanometer, die der Seifenblasenausschläge durch Aichungsblasen von bekanntem Gehalt, unter Controle durch Berechnung nach der Kugelinhaltsformel.

Die beiden Uehnungscurven wurden in folgender Weise ermittelt: Hahn $\mathrm{H}_{1}$ offen; Anfangsspannung $=1 \mathrm{~cm} \mathrm{H}_{2} \mathrm{O}=\mathrm{D}_{1}$ verzeichnet 2 oder 4 isotonische Zuckungen, dam Hahn $H_{1}$ gesehlossen, verzeichnet 1 isometrische Zuckung, Hahn $\mathrm{H}_{1}$ wieder geöffnet und mehrmals einige isotonische Zuckungen, darauf dasselbe Verfahren mit Anfangsspannung

1) Ueber die Theorie und Praxis dieser ron Garten in die physiologische T'echnik eingeführten Mess- und Registrimethode siehe S. Garten: Ueber ein neues Verfahren zur Verzeichnung von Bewegungsvorgängen und seine Anwendung auf den Volumpuls. J'liiger's Arohis. 1904. Bd. 104. S. Bisl. 
$2 \mathrm{~cm} \mathrm{H}_{2} \mathrm{O}=\mathrm{D}_{2}$ u. s. f. Zum Schlusse Rückgang auf $\mathrm{D}_{0}$. Die Controle des Grades der Entleerung bei den einzelnen Anfangsspannungen giebt der Vergleich des Standes $\mathrm{D}_{0}$ mit den systolischen Seifenblasenständen bei den verschiedenen Anfangsspannungen, sind die Punkte $\mathrm{D}_{0}$

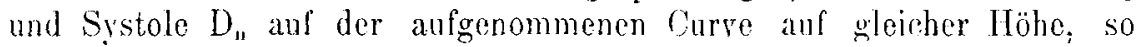
waren Fïllung und Pulsvolum identiseh.

\section{Fig. 2.}

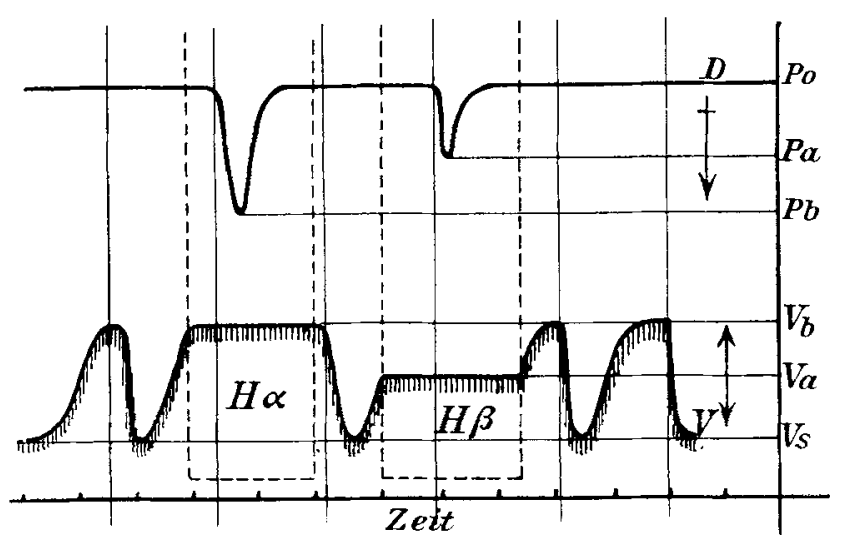

Die Fig. 2 giebt schematisch das Aussehen der auf dem photographischen Papiel verzcichneten Vorgänge. Die obere Curve ist rom Druckhebcl, der sich abwärts bewcgt, gezeichnet, die untere vom tiefsten Punkte der Scifenblase, ihr Curvengipfel ist die maximale Diastole, das Thal die Systole des Herzens, der Vertikalabstand beider (Vb-- Vs) die Differenz der Druckmesser zweier Kugeln, die auf gemeinschaftlichem Fusspunkt aufsitzen $\left(\mathrm{S}_{1}\right.$ und $\mathrm{S}_{2}$ der Fig. 1). Ist Hahn $\mathrm{H}_{1}$ offen, so verzeichnet bloss die Scifenblase, ist er geschlossen, bloss der Druckhebel, dabei giebt der Stand der Seifenblasencurve die Angaben für den Herzinhalt. Ist der Ilahn $\mathrm{H}_{1}$, wic beabsichtigt, im Momente der stärksten Diastole geschlossen worden, so ist der Stand der Blasencurve derselbe wie vorher bei der Diastole unter isotonischer Thätigkeit. Damit hat man die Controle darïber, ob der Hahn $\mathrm{H}_{1}$ im richtigen Iloment geschlossen wurde, denn im anderen Falle würde der Stand des Blasenscheitelpunktes ein tieferer sein (verg]. die Felder $\mathrm{H} \alpha$ und $\mathrm{H} \beta$ der Fig. 2). $\mathrm{H}_{\beta}$ ist Schluss des Hahnes $\mathrm{H}_{1}$ bei unvollendeter Diastole, das Herz arbeitet mit geringerer Füllung, dic isometrische Zuckung fällt niedriger aus.

Anmerkung. Einige der mitgetheilten Versuche, und zwar die mit Antiarinvergiftung, wurden unter constanter, maximaler Füllung bewirkender Anfangsspannung durchgeführt und die Füllungen durch $\Lambda$ bdrehen des Hahns $\mathrm{H}_{1}$ während verschiedener Momente der Diastolo variirt. Dabei bekommt man natürlich bloss die Dehuungscurve der isometrischen Maxima, und zwar nur in Bezug auf die Füllung, nicht aher auf die messhare Anfangsspannung, während die Dehnungscurve der isotonischen Minima nirht zu erkalten ist. 
Zur leichten Feststellung der correspondirenden Punkte der über einander verzeichneten isotonischen und isometrischen Curve habe ich nach S. Garten's') Vorgang durch einen lipikotister Verticallinsen erzeugt, die gleiehzeitig als Zeitmarken gelten können. Ein horizontales Liniensystem in Millimeterabständen lieferte die Graduirung einer Cylinderlinse (s. Garten ibidem).

Die Geschwindigkeit der Zustandsänderung während der Contraction, also die Curvenform, blicb im Laufe der ganzen Untersuchung unberücksichtigt. Lis wurden bloss Maxima und Minima rerzeichnet.

Herrn cand. med. G. Boehm bin ich für freundliche Assistenz zu Dank verpflichtet.

\section{Versuche mit Antiarin.}

1. Versuch vom 1. Mai 1904. Angewandte Giftmenge $0,00001 \mathrm{~g}$. Während des ganzen Versuchs constante Anfangsspannung von $3 \mathrm{~cm}$ $\mathrm{H}_{2} \mathrm{O}$-Druckhöhe. Variation der Füllung durch Schluss von $\mathrm{Hahn}_{1}$ zu verschiedenen Zeiten des Ablaufs der Diastole. Die Vergiftung ist eine tödtliche, zum systolischen Stillstand führende.
a) Normal.
liallungen Dazu Drücke
der isometrisehen Maxima
b) 3 Min. nareh Linbringung des Giftes in das System.
(*) 10 Min. später, kurz nach doc Rhythmushathierung.
d) 5 Min. spiter

\begin{tabular}{|c|c|c|c|c|}
\hline \multirow{3}{*}{$\begin{array}{r}0,05 \\
15\end{array}$} & 0,06 & 0,10 & 0.13 & $0,15 \mathrm{ecm}$ \\
\hline & 18 & 25 & 30 & $32 \mathrm{mmL} \mathrm{lfat}$ \\
\hline & $\begin{array}{r}0,06 \\
13\end{array}$ & $\begin{array}{r}0,10 \\
17\end{array}$ & $\begin{array}{r}0,14 \\
21\end{array}$ & $\begin{array}{l}0,17: \mathrm{cm} \\
\quad 22 \mathrm{~mm} \mathrm{Hg}\end{array}$ \\
\hline 0,01 & 0,08 & 0,09 & 0,10 & $0,12: 0,21 \mathrm{ccm}$ \\
\hline 12 & 17 & 21 & 24 & $25 \mid 25$ Imm 11y \\
\hline 0,08 & 0,09 & 0,14 & 0,20 & $0,21 \mathrm{cem}$ \\
\hline 10 & 15 & 17 & 17 & Intm IJg \\
\hline
\end{tabular}

Fig. 3.

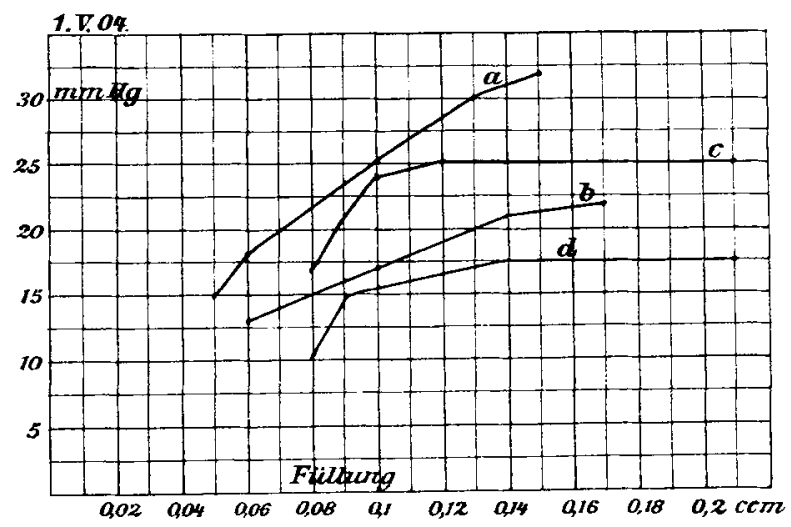

Dehnungscurven der isometrischen Maxima im Verlaufe der Antiarinvergiftung. c und d nach Halbirung.

1) S. Garten, Abhandlungen der Königl. Sächs. Gesellsch. der Wissensch. Math. -phys. Klasse. 1901. Bd, 26. S. 335. 
Resultat: Noch ror Eintritt der Halbirung sinken die isometrischen Haxima gleicher Füllungen. Mit dem Eintritt der Halbirung steigen die isometrischen Maxima im Vergleich zu denen der Vorperiode, erreichen aber nicht die Werthe der Normalperiode. Irnerhalb der Thätigkeit im Halbrhythmus sinken die isometrischen Maxima weiterhin.

Die maximalen Füllungen haben im I aute der oben mitgetheilten Stadien absolut zugenommen (von 0,15-0,20). Später nahmen sie in dem Maasse ab, als der Vergiftungszustand sich dem systolischen Stillstand nähert (ron $0,20-0,05 \mathrm{ccm}$ beobachtet). In Fig. 3 sind die oben unter a-d mitgetheilten Stadien der Vergiftung rraphiseh zusammengestellt, sie zejgt, wie das Sinken der Delnungseurre der isometrisehen Ilaxima (a, b) durch die Halbirung rorübergehend unterbrochen wird (c und d), dabei aber gleichzeitig die absoluten Füllungsmaxima wachsen und zwar anseheinend continuirlich, bis zu dem bei c crreichten llaximum.

2. Versuch rom 30. April 1904. Angewandte Giftmenge 0,000003 $s$ Antiarin (also ea. $1 / 3$ der des vorigen Versuchs, Versuch nit constanter Anfangsspannung. Vergiftung noel tödtich.
a) Normal.
biillungen
der isometrisehen Vaxima
1) : Mlin. narh Vergiftungr
(.) 10 Min. nach Vergiltung (Ilal- birung)
1) 20 Min. naeh Vergiftung

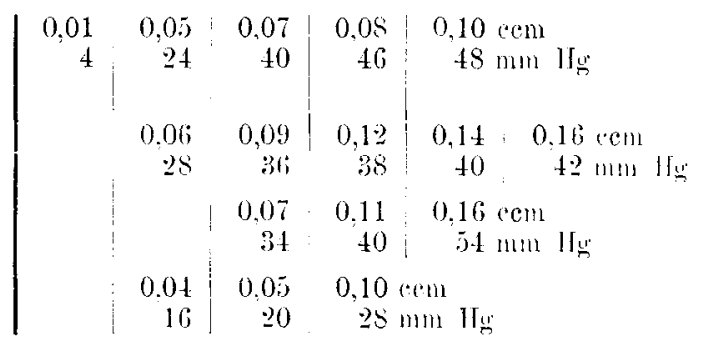

Fig. 4.

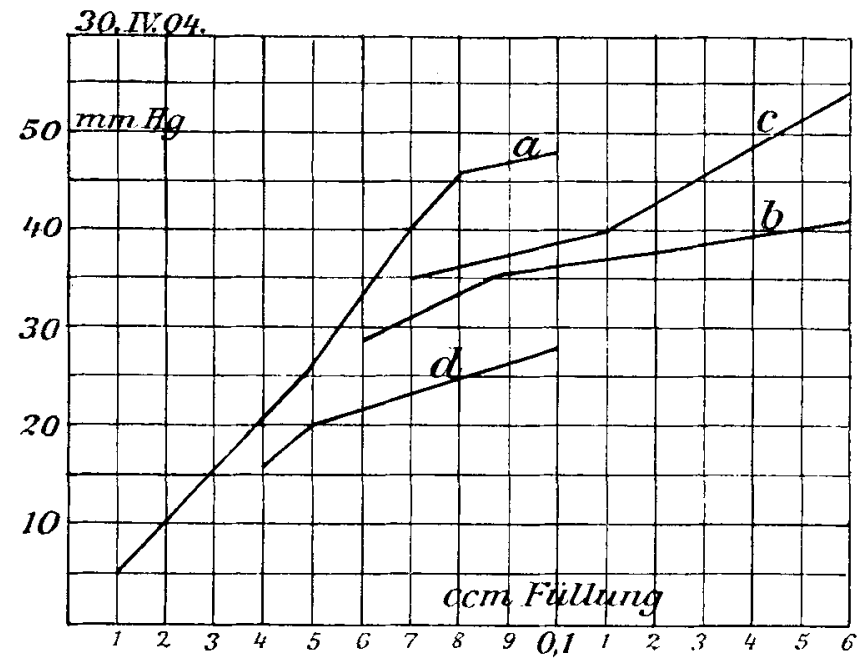

Dehnungscurve der isometrischen Maxima während der Antiarinvergiftungr.

c im Beginn des Halbrhythmus.

Resultat: Die ebenfalls noch tödtlich verlaufende Vergiftung, deren Einzelheiten in Fig. 4 graphisch dargestellt sind, hat bezüglich 
der Dehnungscurven der isometrischen Maxima dicselbe Gesetzmässigkeit, wie bei der dreifach stärkeren Vergiftung erzielt (d. h. Sinken der Dehnungscurve: vergl. a mit $c$ und $d$ ).

Die Pulsvolumina bei constanter Anfangsspannung haben dauernd zugenommen. Dabei ist jedoch in Abschnitt e cine Besonderheit zu Tage getreten: es treten kurz nach der Halbirung höhere, absolute Druckwerthe der isometrischen 7uckung auf, als in der Norm. Die Eüllungen bei denen diese übernormalen Druckwerthe auftreten, sind gleichfalls grösser, als die des Normalzustandes. In der weiteren Verfolgung dieser Erscheinung constatirte ich, dass sic jedesmal zu beobachten ist, wenn man bei sehwachen Vergiftungen, kur\% nach eingetretenem Halbrhythmus, dic Curven aufnimmt. Sic ist ferner nur bei den Füllungsgraden rorhanden, die absolut grösser sind, als die maximalen der Normalperiode. In der Fig. 5 sind zwei Momente aus einem zur Erforschung der Bedingungen des Phänomens angestellten Versuch abgebildet. Die obere Curve ist rom Druckhebel geschrieben, die untere rom Scheitelpunkt der

lig. 5.

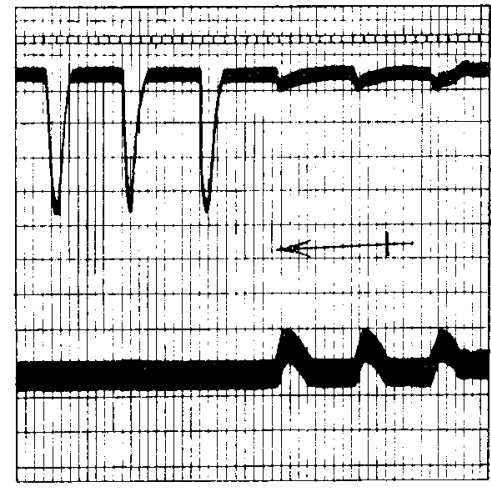

Halbrhythmus.

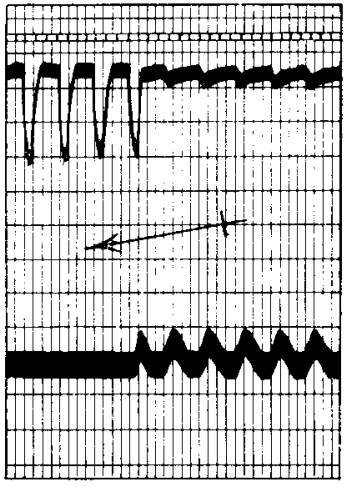

Normal.

'Zunahme der isometrischen Maxima gleichen Füllungsdruckes bei Zunahme der Füllung im llalbrhythmus.

Seifenblase. (Nlan sieht, wie im Halbrhythmus die Volumina grösser sind, wie vorher, und gleichzeitig die zugehörigen Drucke wachson.) Bei den Füllungszunahmen, die vor der Halbirung schon im Normalrhythmus auftreten, ist die Erseheinung nicht zu finden. Dic Bedingungen des Zustandekommens sind also Pausenvergrösserung und Füllungszuwachs und die Erscheinung direkt ejne Folge der Halbirung und erst indirekt eine der Vergiftung.

Die folgenden Versuche sind unter Variation der Anfangsspannung mit Hülfe des Gefüsses D (Fig. 1) angestellt, ergeben also auch die Dehnungscurren der isotonischen Minima.

Versuch 3 rom 6. Yai 1904. Angewandte Giftmenge 0,000006 g Intiarin. 


\begin{tabular}{|c|c|c|c|c|c|c|}
\hline Anfangsspannung & 1 & 2 & $:$ & 4 & 5 & $6 \mathrm{~cm} \mathrm{H}_{2} \mathrm{O}$ \\
\hline $\begin{array}{l}\text { a) Normalzustand. } \\
\left.\text { Diastolisehe Fiullung }{ }^{2}\right) \\
\text { Druck }\end{array}$ & $\begin{array}{r}0,01 \\
2,5\end{array}$ & 0,02 & $\begin{array}{r}0,03 \\
9\end{array}$ & $\begin{array}{r}0,05 \\
14\end{array}$ & $\begin{array}{r}0,06 \\
16\end{array}$ & $\begin{array}{l}0,07 \\
17\end{array}$ \\
\hline b) 1 Min. nach Vergiftung & $\begin{array}{r}0,01 \\
3\end{array}$ & & $\begin{array}{r}0,03 \\
7\end{array}$ & $\begin{array}{r}0,05 \\
12\end{array}$ & $\begin{array}{r}0,06 \\
15\end{array}$ & $\begin{array}{r}0,08 \\
18\end{array}$ \\
\hline c) 3 Min. später & $\begin{array}{r}0,02 \\
4\end{array}$ & $\begin{array}{r}0,05 \\
8,5\end{array}$ & $\begin{array}{r}0,06 \\
10\end{array}$ & $\begin{array}{r}0,07 \\
11\end{array}$ & $\begin{array}{r}0,0 S \\
12\end{array}$ & $\begin{array}{r}0,10 \\
17\end{array}$ \\
\hline d) 3 Min. später & $\begin{array}{r}0,02 \\
3\end{array}$ & $\begin{array}{r}0,04 \\
5\end{array}$ & $0,0 \%$ & $\begin{array}{r}0,08 \\
10\end{array}$ & & $\begin{array}{r}0,10 \\
10\end{array}$ \\
\hline $\begin{array}{l}\text { e) } 3 \text { Min. später. } \\
\text { Ufalbirung vollendet. }\end{array}$ & $\begin{array}{r}0,02 \\
5\end{array}$ & $\begin{array}{r}0,08 \\
11\end{array}$ & $\begin{array}{r}0,10 \\
15\end{array}$ & $\begin{array}{r}0,12 \\
18\end{array}$ & $\begin{array}{r}0,12 \\
16\end{array}$ & 0,12 \\
\hline 1) 4 Min. später & $\begin{array}{r}0,03 \\
6\end{array}$ & $\begin{array}{r}0,06 \\
11\end{array}$ & $\begin{array}{r}0,10 \\
15\end{array}$ & $\begin{array}{r}0,11 \\
17\end{array}$ & $\begin{array}{r}0,12 \\
18\end{array}$ & 0,12 \\
\hline
\end{tabular}

Resultat: Die Dehnungscurve der isotonischen Minima sinkt im Laufe der Vergiftung in der angewandten Belastungsbreite von $0-6 \mathrm{~cm}$ $\mathrm{H}_{2} \mathrm{O}$ dauernd bis zu einem Maximum. Die Dehnungscurve der isometrischen Maxima verhiilt sich wie früher; Abschnitt c, 4 zeigt die bei dem vorigen Versuch 2 discutirte absolute Druckzunahme auf übernormale Werthe.

In diesem Versuch wurde das Herz nach Aufnahme der Reihe $f$ noch $2 \frac{1}{2}$ Stunden lang unter der Anfangsspannung von $4 \mathrm{~cm} \mathrm{H}_{2} \mathrm{O}$ beobachtet, dabei war schliesslich das Verhältniss $\frac{\text { Druck }}{\text { Volum }}=\frac{16}{0.10}$ gegen $\frac{17}{0.11}$ bei der gleichen Anfangsspannung des Zustandes $\mathrm{f}$ in oben-

Fig. 6a.

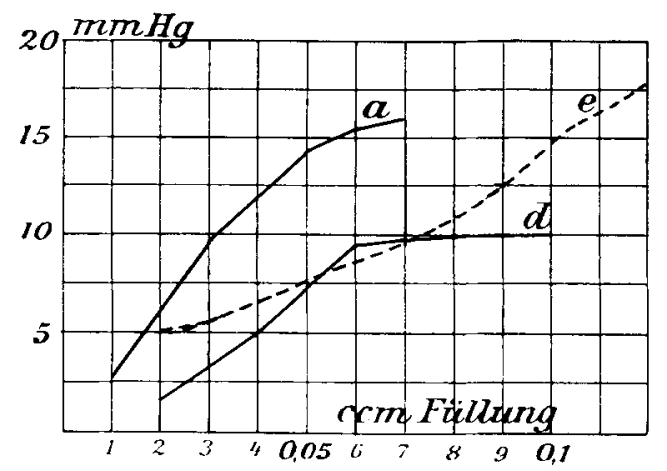

Dehnungscurve der isometrischen Maxima der Stadien. a, d, e des Versuchs 3.

a im Halbrhythmus.

1) Bezüglich der Gesetzmässigkeit dieser Abnahme der Maxima bei wachsenden Anfangsspannungen, aber constanter Füllung, siehe O. Frank, Dynamik, S. 10 des Sep.-Abdr.

2) Für den Belastungsgrad identisch mit dem Pulsvolum. 
liig. 6 b.

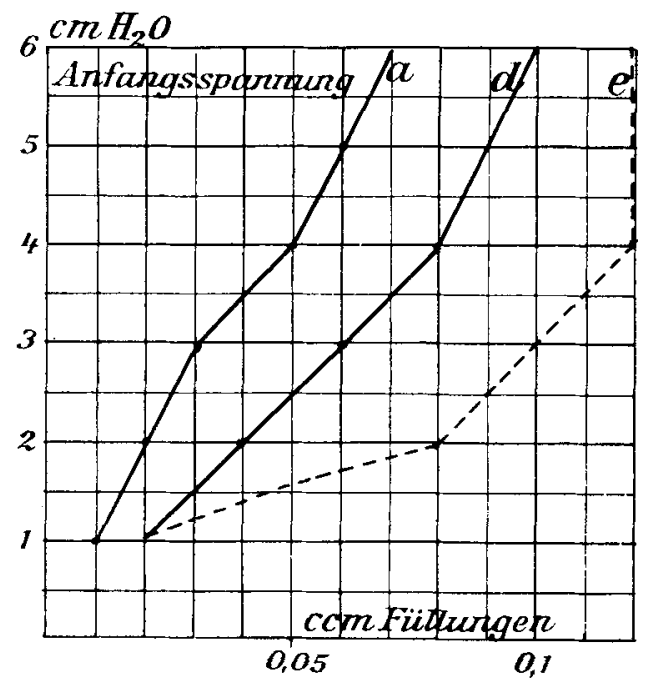

Dehnungscurve der isotonischen Minima der Stadien, a, d, e des Versuchs 3. c im Halbrhythmus.

stehonder Tabelle. Es dürfte demnach angenommen werden, dass der durch die Werthe: Halbrhythmus, Druck $=\frac{16}{0.10}$, Anfangsspannung $4 \mathrm{~cm} \mathrm{H}_{2} \mathrm{O}$ definirte Zustand des Herzventrikels, der Endzustand ist, dem die chemischen Vorgänge im System Ventrikel $+5,0 \mathrm{ccm}$ Ringer'sche Lösung zustrebten, nachdem 0,000006 g Antiarin in das System gebracht worden waren.

Der nächste Versuch wurde mit einer noch kleineren Dosis von $0,0000025 \mathrm{~g}$ Antiarin angestellt und die Dehnungscurven bloss im Normalrhythmus festgestellt.

Versuch 4, vom 8. Mai 1904. 0,0000025 g Antiarin.

\begin{tabular}{l|r|r|r|r|r|r}
\hline \multicolumn{1}{r|}{ Anfangsspannung } & 1 & 2 & 3 & 4 & 5 & $6 \mathrm{~cm} \mathrm{H}_{2} \mathrm{O}$ \\
\hline a) Normal 4 Uhr 55 Nir. Volum & 0,02 & 0,04 & 0,07 & 0,11 & 0,16 & 0,20 \\
& 3 & 5 & 10 & 17 & 20 & 20 \\
b) 5ruck & 0,01 & 0,02 & 0,04 & 0,05 & 0,09 & 0,16 \\
(a) 5 Uhr 7 Min. (1 Min. nach 18 Min. & 3 & 6 & 10 & 13 & 20 & 30 \\
Tergifting) & 0,02 & 0,07 & 0,10 & 0,15 & 0,16 & 0,19 \\
& 3 & 8 & 13 & 16 & 9 & 14
\end{tabular}

Resultat: Der Versuch giebt in seiner Abtheilung b ein principiell neues Resultat: Verminderung der Füllung gleicher Anfangsspannung unter gleichzeitiger Vergrösserung der isometrischen Maxima, also eine positive Wirkung. Vergl. die graphische Darstellung Fig. $7 \mathrm{a}$ und b. Die weitere spezielle Verfolgung der Erscheinung ergab, dass sie eintritt, wenn man mit Giftdosen arbeitet, die 
eben gerade für die Herbeiführung des Halbrhythmus ausreichen und den Ganzrhythmus verhältnissmässig lange bestehen lassen, dann zeigt sich die Erscheinung als erstes Stadium der Vergiftung im Ganzrbythmus, vielleicht lässt sie sich dureh noch weiteres Herabgehen mit den Dosen auch als einzige und bleibende Wirkung des Giftes herbeiführen, doch habe ich daribler keine Versuche angestellt.

Fig. Ta.

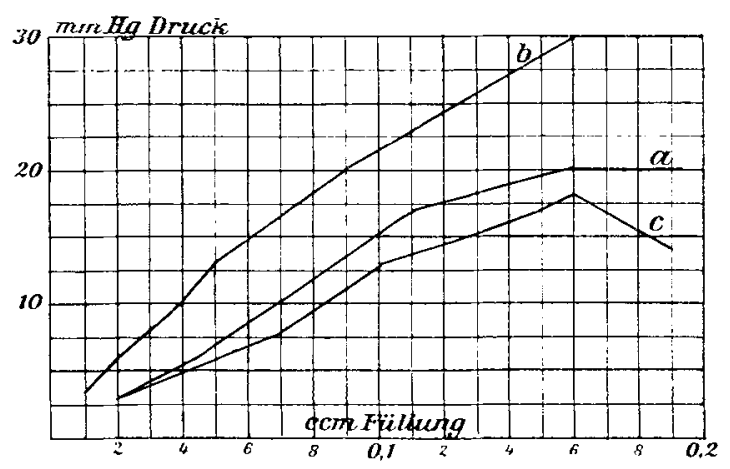

Dehnungseurven der isometriselen Maxima.

Fig. $7 \mathrm{~b}$.

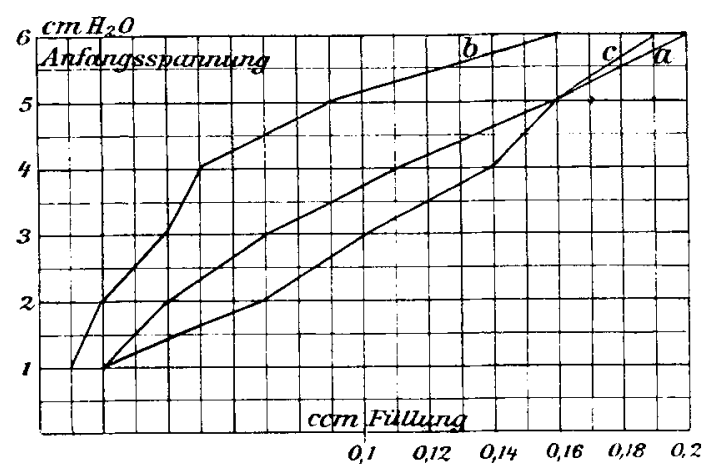

Dehnungscurven der isotonischen Minima. Versuch 4.

Gegen die mögliche Annahme, dass bei der nach Einbringung des Giftes nöthigen Neufeststellung der Anfangsspannung O, versehentlich ein negativer Druck hergestellt, würde, spricht, abgesehen von der Constanz der Erscheinung, schon der Umstand, dass die isometrischen Drucke über die Normalwerthe der gleichen Füllung steigen.

Die Erscheinung ist eine Ausnahme von den Frank'schen Gesetzen, eine Sache für sich. Nach diesen war zu erwarten, dass der verminderten Herzfüllung ein gleichfalls vermindertes Maximum der isometrischen Zuckung zukäme; dann hätte es sich um einzige Wirkung auf die Elasticitït gehandelt; ich möchte diese an der Energieentwicklung sich abspielende Wirkung eine "rein positive" nennen, zum Lnterschied 
Dynamik des Froschherzventrikels bei nicht tödtlicher Digitalisvergiftung. 501 von der ohen für den II albrhythmus constatirten Erseheinung, die als Folge der vermehrten Füllung sich erklärt und dic man vielleicht "pscudopositive Wirkung" nennen könnte.

\section{Versuche mit Helleborein.}

1. Versueh vom 5. Mai 1904 . Giftmenge $0,0002 \mathrm{~g}$.

\begin{tabular}{|c|c|c|c|c|c|}
\hline Infangsspannung & 1 & 2 & 3 & 4 & $5 \mathrm{~cm} 110$ \\
\hline $\begin{array}{l}11 \text { Chr 25 MLin. normal. Fïllung } \\
\text { Druck }\end{array}$ & $\begin{array}{r}0,005 \\
4\end{array}$ & $\begin{array}{r}0,08 \\
10\end{array}$ & $\begin{array}{r}0,14 \\
20\end{array}$ & $\begin{array}{r}0,16 \\
23\end{array}$ & - \\
\hline $\begin{array}{l}\text { 12 Shr } 04 \text { Min. } 29 \text { Min. nach } \\
\text { Vergiftung }\end{array}$ & - & $\begin{array}{r}0,07 \\
5\end{array}$ & 0,15 & $\begin{array}{r}0,18 \\
10\end{array}$ & - \\
\hline $\begin{array}{l}\text { 12 Ihr } 18 \text { Min. } \\
\text { nach der llatbirung }\end{array}$ & 0.06 & $\begin{array}{r}0,15 \\
12\end{array}$ & $\begin{array}{r}0,17 \\
15\end{array}$ & $\begin{array}{r}0,20 \\
18\end{array}$ & $\begin{array}{r}0,21 \\
18\end{array}$ \\
\hline 12 lhr $30 \mathrm{Min}$ & $\begin{array}{r}0.04 \\
5\end{array}$ & $\begin{array}{r}0,10 \\
10\end{array}$ & $\begin{array}{r}0,18 \\
14\end{array}$ & $\begin{array}{r}0,21 \\
16\end{array}$ & $\begin{array}{r}0,23 \\
18\end{array}$ \\
\hline
\end{tabular}

Resultat: I)ie Vergiftung verlief nicht tödtlich, das Präparat wurde noch 3 Stunden lang beobachtet. Dic Ėscheinungen nach der Vergiftung sind analog den bei der Antiarinvergil'tung aufgetretenen, d. h. es trat Zunahme der Füllungen gleicher Anfangsspannung unter Abnahme der zugehörigen Gipfel der isometrischen Haxima auf. Dic Halbirung brachte die schon für Antiarin constatirte Erhöhung der Dehnungseurve der isometrischen Ilaxima (pseudopositive Wirkung).

2. Versuch vom 4. Januar 1905. 0,0006 g Helleborein.

\begin{tabular}{|c|c|c|c|c|}
\hline Iufangsspannumgen & 1 & 2 & 3 & 4 \\
\hline $\begin{array}{r}11 \text { Uhr } 55 \text { Min. normal. } \\
\text { Fîllumigen } \\
\text { Druicko }\end{array}$ & $\begin{array}{r}0,03 \\
8\end{array}$ & $\begin{array}{r}0,13 \\
14\end{array}$ & $\begin{array}{r}0,17 \\
21\end{array}$ & $\begin{array}{r}0,20 \\
32\end{array}$ \\
\hline 12 l'hr nach Jalbirung & $\begin{array}{r}0,10 \\
13\end{array}$ & $\begin{array}{r}0,22 \\
32\end{array}$ & $\begin{array}{r}0,33 \\
36\end{array}$ & $\begin{array}{r}0,38 \\
37\end{array}$ \\
\hline 12 Lhe 30 Mlin. & $\begin{array}{r}0,11 \\
20\end{array}$ & $\begin{array}{r}0,25 \\
30\end{array}$ & $\begin{array}{r}0,32 \\
35\end{array}$ & $\begin{array}{r}0,4 \% \\
34\end{array}$ \\
\hline
\end{tabular}

Resultat: Wic im vorigen Versuch; besonders deutliche Zunahme der Dehnungseurve der isometrischen Jaxima nach der Halbirung. Keine tödtliche Vergiftung auf Grund 3stündiger weiterer Beobachtung.

3. Versuch vom 5. Januar 1905. 0,0005 g Helleborein.

\begin{tabular}{|c|c|c|c|c|c|}
\hline Antangsspannuny" & 1 & 2 & 3 & 4 & 5 \\
\hline $\begin{array}{r}\text { a) } 4 \text { Uhr } 48 \text { Min. numal. } \\
\qquad \begin{array}{l}\text { lïllumg } \\
\text { l)ruek }\end{array}\end{array}$ & $\begin{array}{r}0.03 \\
3\end{array}$ & $\begin{array}{r}0,06 \\
4\end{array}$ & $\begin{array}{r}0,10 \\
5\end{array}$ & $\begin{array}{r}0,13 \\
6\end{array}$ & $\begin{array}{l}0,18 \mathrm{cem} \\
7 \mathrm{~mm} 1 \mathrm{gg}\end{array}$ \\
\hline $\begin{array}{l}\text { b) } 4 \text { Uhr } 55 \text { Min.. } 1 \text { Nin. } \\
\text { nach Vergiftung }\end{array}$ & 0,02 & 0,05 & $\begin{array}{r}0,09 \\
10\end{array}$ & $\begin{array}{r}0,12 \\
12\end{array}$ & $\begin{array}{r}0,15 \\
13\end{array}$ \\
\hline c) 6 Uhr $30 \mathrm{Min}$ & $\begin{array}{r}0,05 \\
3\end{array}$ & - & $\begin{array}{r}0.11 \\
6\end{array}$ & - & $\frac{0,20}{7}$ \\
\hline
\end{tabular}


Resultat: Im Stadium b kommt hier die lür die Antiarinvergiftung constatirte rein-positive Wirkung zur Beobachtung. (Zunahme der isometrischen Drucke, trotz Abnahme der Füllungen gleicher Anfangsspannungen.)

Es bestehen also in der Wirkung des Antiarins und Helleborcins keine Unterschiede der Art, sondern nur solche des Grades. Die mitgetheilten Versuche, die sich mit vielen anderen decken, zeigen, dass bei einer zur Hervorbringung eines bestimmten Effektes (Halbrhy thmus, systolischer Stillstand) durch ihr absolutes Gewicht befähigten Giftmenge der Verlauf der Vergiftung ein viel langsamerer ist, was nach den Eingangs erörterten chemischen Vorstellungen über den Vergiftungsverlauf einer geringeren Reactionsgeschwindigkeit entspräche. Nebenbei sind die absoluten Dosen bei Helleborein grösser als bei Antiarin. Speciell ist noch zu bemerken, dass die Zunahme der isometrischen Maxima im Halbrhythmus über die Werthe der Norm (pseudopositive Wirkung) bei der Helleboreinvergiftung besonders leicht und lange zu beobachten ist.

\section{IIr. Digitalin.}

Die mit Digitalin ${ }^{1}$ ) angestellten Versuche verliefen ganz conform den Helleboreïnersuchen, so dass ich auf detaillirte Wiedergabe verzichten kann.

\section{Strophantin.}

Versuche vom 17. Januar 1905 mit $0,0003 \mathrm{~g}$ Strophantin.

\begin{tabular}{|c|c|c|c|c|}
\hline Anfangsspannung & 1 & 2 & 3 & $4 \mathrm{~cm} \mathrm{H} \mathrm{H}_{2}(1$ \\
\hline $\begin{array}{r}\text { a) } 5 \text { Uhr } 25 \text { Min. normal. } \\
\text { Volum } \\
\text { Druck }\end{array}$ & $\begin{array}{r}0,07 \\
13\end{array}$ & $\begin{array}{r}0,15 \\
24\end{array}$ & $\begin{array}{r}0,19 \\
28\end{array}$ & $\begin{array}{l}0,24 \mathrm{~cm} \\
25 \mathrm{~mm} \mathrm{H} \mathrm{H}_{\mathrm{g}}\end{array}$ \\
\hline $\begin{array}{l}\text { b) 5 Lhr } 33 \text { Min. unmittel- } \\
\text { bar nach Vergiftung }\end{array}$ & $\begin{array}{r}0,03 \\
4\end{array}$ & $\begin{array}{r}0,10 \\
20\end{array}$ & $\begin{array}{r}0,14 \\
32\end{array}$ & $\begin{array}{r}0,20 \\
34\end{array}$ \\
\hline c) 5 I Thr 37 Min. & $\begin{array}{r}0,03 \\
4\end{array}$ & & $\begin{array}{r}0,08 \\
21\end{array}$ & $\begin{array}{r}0,13 \\
30\end{array}$ \\
\hline d) 5 Uhr 53 Min. & $\begin{array}{r}0,05 \\
5\end{array}$ & $\begin{array}{r}0,10 \\
20\end{array}$ & $\begin{array}{r}0,14 \\
25\end{array}$ & $\begin{array}{r}0,17 \\
27\end{array}$ \\
\hline e) 6 Ülr 9 MTin. & $\begin{array}{r}0,03 \\
3\end{array}$ & $\begin{array}{r}0,10 \\
10\end{array}$ & $\begin{array}{r}0,18 \\
\quad \geqslant 0\end{array}$ & $\begin{array}{r}0,23 \\
23\end{array}$ \\
\hline f) 6 Uhr 20 Min. & 0,03 & $\begin{array}{r}0.10 \\
7\end{array}$ & $\begin{array}{r}0,18 \\
17\end{array}$ & $\begin{array}{r}0,23 \\
19\end{array}$ \\
\hline $\begin{aligned} \text { g) } 6 \text { Uhr } & 40 \text { Xlin. nach dor } \\
& \text { Halbirung }\end{aligned}$ & $\begin{array}{r}0,12 \\
20\end{array}$ & $\begin{array}{r}0,20 \\
25\end{array}$ & $\begin{array}{r}0,25 \\
25\end{array}$ & $\begin{array}{r}0,28 \\
24\end{array}$ \\
\hline
\end{tabular}

Auch hier ist das Resultat conform dem mit den anderen Glykosiden erziclten; zunächst eine rein positive Wirkung, dann Abnahme der isometrischen Maxima gleicher Füllung, die durch dic Halbirung gebessert

1) Es wurde ein ron Prof. Kiliani isolirtes Digitalein, das mir Prof. Boebm zur Verfügung stellte, benutzt. Das Präparat ist noch liein ganz einheitlicher Körper, aber jedenfalls viel reiner, als die als Digitalin im Handel befindlichen Gomenge. 
Dynamik des Froschherzentrikels bei nicht tödticher Digitalisvergiftung. 50.3

Fig. $8 \mathrm{a}$.

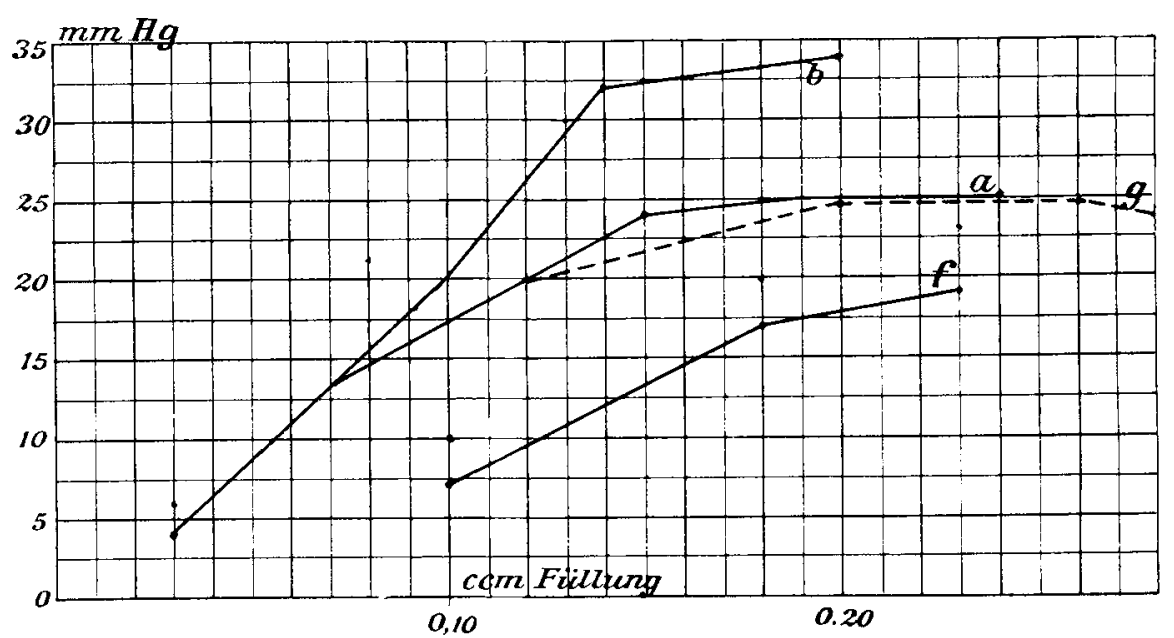

Dehnungscurve der isometrischen Maxima. Strophantinversuch.

Fig. $8 \mathrm{~b}$.

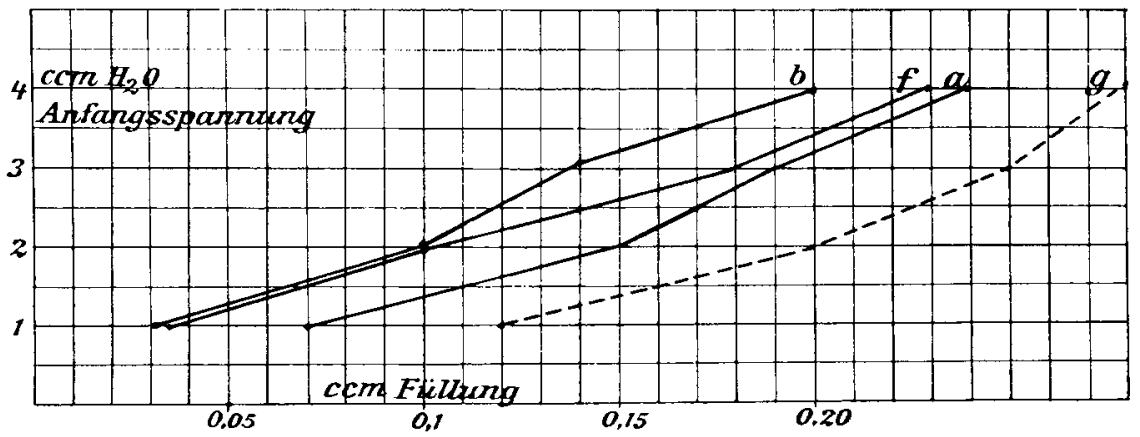

Dehnungscurve der isotonischen Minima. Strophantinversuch.

Fig. 9.

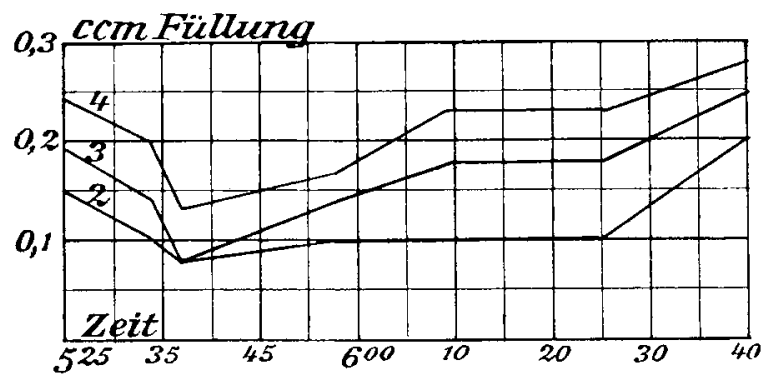

Strophantinversuch. Aenderungen der Pulsvolumina gleicher Anfangsspannungen $\left(2,3,4 \mathrm{~cm} \mathrm{H} \mathrm{H}_{2} \mathrm{O}\right)$ nach der Zeit. 
wird. Vergleiche dic graphische Darstellung Fig. 8a u. b. Dic Fig. ? giebt die zeitliche Aenderung der Pulsvolumina gleicher Anfangsspannungen und zeigt, wie die diastolisehen Füllungen gleicher Drucke Anfangs sinken und später abnorm gross werden ${ }^{1}$.

\section{Zusammeníasung der Resultate.}

Nit den untersuchten Digitalisglykosiden, Antiarin, Helloboreü, Digitalin und Strophanthin Jassen sich willkürlich folgcode Erscheinungen herbeiführen und experimentell beherrschen:

1. Abnahme der Füllungen gleicher Anfangsspannungen unter gleichzeitiger Zunahme der Druckmaxima der correspondirenden isometrischen Zuckungen (rein positive Wirkung). Vielleicht kann man in der Erseheinung das Analogon der gefisssverengernden Wirkung sehen. Der positive Effekt ist jedenfalls ein allgemeines Phänomen, denn ich konnte früher nachweisen, dass auch die Erregbarkeit des Ventrikels unter Digitaliswirkung in den ersten Stadien der Vergiftung gesteigert ist. (Arch. f. exp. Path. u. Pharmak. Bd. 45. Taf. III. Fig. 7 A.)

2. Zunahme der Füllungen gleicher Anfangsspannungen (wie schon von Williams ${ }^{2}$ ) constatirt) unter glcichzeitiger Abrahme der correspondirenden Maxima der isometrischen Zuckung (negative Wirkung).

3. Eine Besonderheit gilt für den Halbrhythmus, indem die bei der isometrischen Zuckung erzielten Ilaxima über dic der vorhergchenden Stadien des Ganzrhythmus, bei gecigneter Dosirung auch über die des Normalzustandes, unter Vergrösserung der Füllungen gleicher $\Lambda$ nfangsspannung wachsen ${ }^{3}$ ) (pseudopositive Wirkung). Die lischeinung orklärt sich aus dem allgemein physiologischen Verhalten des Herzmuskels ${ }^{4}$ ), der Rhythmusverlangsamung innerhalb gewisser Grenzen mit Erhöhung der Einzelzuckung beantwortet. (Pausengesetz, Bowditseh, F. B. Hofmann, l. e.)

4. Eine Folge der sub 3 angeführten Besonderheit ist es, dass in einem solehen Stadium der Verlaul der Dehnungscurve der isometrischen Maxima mit dem der analogen Curre des Normalzustandes sich deckt (Fig. 8a) insofern, als bis zum Füllungsmaximum des Normalzustandes im Halbrhythmus, jedem Fülungswerth der Druckwerth des normalen entspricht.

5. Die sub 2 und 3 genannten Erseheinungen lassen sich willkürlich als Stationärzustand durch Dosirung herbeiführen.

1) Ich wollte auch das Bigitoxin in den Kreis der Untersuchung ziehen, besonders weil es in den später zu erwähneuden Versuchen ron Gottlieb und Magnus eine Rolle spielt, indess ist seine Wasserlöslichleit so gering, dass ich für meine Zwecke keine Lösung mit bekanntem Gehalt herstellen lionnte.

2) Arch. f. exp. Path. 1. Phatm. BA. 13. S. 8.

3) Wobei es dahingestellt bleiben muss, ob dem gleichen lüllungszuwachs am normalen Ventrikel nicht vielleicht ein noelı grösserer Druckzuwachs entsprochen bätte.

4) Wahrscheinlich überhaupt aller irritablen Substanzen; rgl. F. B. Hofmann, der dasselbe Verhalten fïr die motorische Nervenendigung nachwies. Pllïger's Arch. 1904. Bd. 103. S. 291. 
6. Bei der für die Entersuchung gewählten Breite der Anfangsspannungen von $0-5 \mathrm{~cm} \mathrm{H}_{2}(\mathrm{O}$ ist das Pulsvolum gleich der Füllung; die "Dchnungscurve der isotonischen Maxima" also nicht gesunken.

\section{Dic Untorsuchungen von O. Frank.}

Die Dynamik des Ventrikets des Froschherzens ist ron O. Frank als crste Nutzanwendung der von ihm ausgearbeiteten Technik der Dynamik des Normalzustandes untersucht worden. Frank kam zu folgenden Resultaten:

In demjenigen . Stadium der Helleboreinvergiftung, in dem cine Verlangsamung des Herzschlages bereits eingetreten ist, ohne dass die Regelmässigkeit der Schlagfolge gestört ist,

50. bleibt die Dehnungscurve der isometrisehen Vlaxima im grossen Ganzen unverändert. Möglicherweise werden dic Naxima etwas erhöht.

53. Dio Dehnungseurve der isotonischen Mlinima wird nur für den Theil verïndert (erniedrigt), bei dem die linzelcurven nicht dic horizontale Strecke im Minimum aufweisen, also nur bei den niederen Drueken.

63. Dic Veränderungen der Arbeit bezw. des Effectes durch Digitalis lassen sich unmittelbar aus den Sätzen 50,54 ableiten. Bei der natürlichen Zuckung des Herzmuskels kann cine Erhöhung der Arbeit der cinzelnen Zuckung nur für die niedrigen Anfangsdrucke eintreten.

64. Bei den geringsten Anfangsdrurken kann die Erniedrigung des Ilinimums der Zuckungseurve so weit gehen, dass trota der Vor angsamung eine lirhöhung des mittleren liffects stattfindet."

Unter der, wie mir scheint, berechtigten Voraussetzung, dass Frank's Untersuchungen an dem im Halbrhythmus thätigen, mit Helleborën vergifteten Herzen angestellt sind, ergiebt sich völlige Uebereinstimmung zwisehen Frank's und meinen Resultaten. In diesem Stadium ist die Dehnungseurve der isometrischen Maxima normal, d. h. den einzelnen Füllungen entsprechen die isometrischen Druckwerthe der Normalperiode, die Füllungen (und Pulsvolumina) sind aber abnorm gross (nach Frank (53) ist die Dehnungscurve der isolonischen Minima gesunken).

Es besteht aber nach meinen Beobachtungen die Besonderheit, dass die Dehnungscurve der isometrischen Maxima wieder normal ist. Bestände in diesem, durch ein gewisses chemisches Gleichgcwicht definirbaren Vergiftungszustande Ganzrhythmus, so wäre auch hier die Dehnungscurve gesunken. Allgemein sinkt also diese Dehnungscurve, speciell aber kann diese negative Becinflussung durch einen ganz anderen Vorgang (Halbirung) compensirt werden; es ist sogar möglich, dass sie übercompensirt wird, dies besonders dann, wenn der Ausgangsrhythmus cinen gewissen Grenzwerth der Frequenz nach unten nicht übcrschreitet, d. i. nicht abnorm langsam ist. Der Widerspruch zwischen den Ergebnissen der Frank'schen Untersuchungen und den meinigen ist demnach nur ein scheinbarer und dürtte sich durch Berücksichtigung der Frequenzänderung im Halbrhythmus und deren allgemein physiologischen Consequenzen beseitigen Jassen.

Der ganze Bereich aller möglichen Fälle der Herzmechanik, wie er durch Frank's Jiagramm, Fig. 1, S. 3 d. Sep.-Abdr. gegeben ist, kommt für die Beurtheilung der Wirkung der Digitaliskörper nicht in 
Betracht, vielmehr nur jener allerkleinste Thej, der durch dic im Oryanismus möglichen Anfangsspannungen definirt ist, dessen oberste Grenze demnach durch die absolute Kraft des Vorhofes gesteckt ist. Nach Frank (Dynamik, S. 25 (l. Sep.-Mhdr.) ist das etwa 5-10 mm Hg. ${ }^{1}$ ).

Bedeutung der beobachteten Thatsachen für den Kreislaul.

Die aus den vorliegenden Beobachtungsthatsachen ableitbaren unmittelbaren Folgen der Wirkung der Digitaliskörper für den Kreislauf. müssen sich am Pulsvolum resp. der Strömungsgeschwindigkeit des Blutes in den Gefässen äussern. Von diesem Gesichtspunkte aus betrachtet, kann der beobachteten ersten, rein-positiven Wirkung (Verkleinerung des Pulsvolums und Erhöhung der isometrischen Maxima) für die Norm ${ }^{2}$ ) keine Bedeutung zukommen, dem folgenden Stadium (Vergrösserung des Pulsvolums unterVerminderung der isometrischen Maxima im Ganzrhythmus) kann unter gewissen Bedingungen eine Zunahmo der arteriellen Strömungsgeschwindigkeit zu verdanken scin. Dies muss cintreten, wenn die Füllungen grleicher Anfangsspannungen schon rergrössert sind (die Dehnungsearve der isotonischen Minima gesunken ist) zu einer Zeit, wo noch kein oder nur ein unbedentender negativer Sinfluss auf die Druckkraftentwicklung (Dehnungseurve der isometrischen Maxima) stattgefunden lat ${ }^{3}$. Die isometrische 7uekung ist methaniseh der obere Grenzfall der Druckleistung des Ventrikels; wem der Grad der Belastung (der Blutdruck), mit der die natürliche Zuckung in ihrer isotonischen Componente (s. o.) verläuft, schon in der Norm wesentlich kleiner ist, als die maximal entwickelbaren Druckkräfte, so können diese noch ojne Verminderung ertragen, ohne praktisch schädigende Kreislaufsfolgen zu äussern. Dann kann allein dureh die zunchmende diastolische Dehnbarkeit (Sinken der Dehnungscurve der isotonisehen Ilinima) schon im Ganzrhythmus eine Geschwindigkeitszunahme des Blutstroms crzielt werden. Die Beobachtungsthatsache, dass am gefensterten Froseh in diesem Stadium der Vergiltung der Ventukel sich ebenso vollständig contrahirt wie in der Norm, spricht a priori für die obige Auflassung, für die nooh ganz allgemein geltend zu machen ist, dass der Organismus immer mit Ueberschuss arbeitet.

Der bei stärkeren Vergiftungen aus anderen Gründen (Erregbarkeitsabnalıme) eintretende Halbrhythmus muss in seinen willkürlich herbeiführbaren Anfangsstadien keinen unbedingten fördernden Liufluss auf die Strömungsesehwindigkeit (und damit den Blutdruck) haben; einen be-

1) Auch dieser Werth, der ja einer isumetrisehen Luekung entnommen ist, ist noch riel zu hoch. Ich fand in meinen Versuchen, dass im Allgemeinen schon ron $5 \mathrm{~cm}$ II.2O Anfingsspannung ab kein Zuwachs der Ventrikelfïllong mehe erfolgt. facobj maass währent der l'hätiglicit des Vorhofes noch geringere bruckwerthe. Areh. P. exp. l'ath. w. l'harm. 4t. 1900.)

2) Wialnend der Erstheinung unter den pathologischen lireislanlsbedingungen der Aortenstenose praktische Bedeutung zulommen künnte.

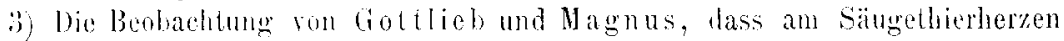
bei constanter Anfangsspamung (nicht Jiillung) durch l)igitalis dio Maxina der isometrischen linzelzuckungen wachsen, liisst sich viclleicht dafür greltend machen. 
dingten aber dann, wenn das P'ulsrolum einer Systole des Halbrhythmus grösser ist als das zweier im normalen Ganzrhythmus.

Bei dieser Betrachtmng int von dor Gefässwirkung der Digitaliskörper abgesehen und rorausgesetzt, dass das Pulswolum auch grösser werden kann, d. h. genügend Blut dem Herzen zuströmt.

lig. 10.

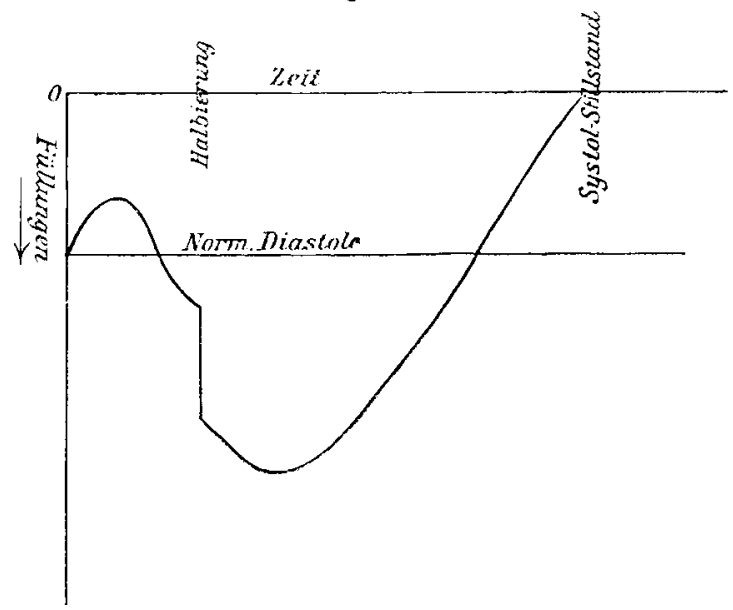

In der beistehenden Fig. 10 habe ich versucht, die Aenderung der diastolischen Ventrikellüllungen im Laufe einer continuirlich zunehmenden Vergiltung nach der Zeit dat\%ustellen. Zunäehst nehmen die diastolischen Füllungen gleicher Anfangsspannung unter dic Xorm ab, steigen dann auf abnorm hohe Werthe, deren Betrag durch den eintretenden Halbrhythmus plötzlich vergrössert wird, aueh im Halbrhythmus ist dio Tendenz zur Vergrösserung der Füllung noch zu bemerken; am unteren Lmkehrpunkt der Curve setzt dann der Process ein, der sich als Schrumplung äussert und mit dem systolischen Stillstand endigt.

\section{blutdruekversuche.}

In zahlreichen Versuchen habe ich am Froseh das Verhalten des Blutdrueks, also die Aenssermg der Strömungsgeschwindigkeit, im Laufo von Vergiftungen untersucht, deren dbauf ich aus den Versuchon am ausgesehnittenen Herzen beurtheiten lsonnte. Da es mir dabei auf den ganzen Verlauf der Vergiftung ankam, mussten sich die Versuche meist über mehrere Tage crstrecken, was bei Frischen auf keine technisehen Schwierigkeiten stösst.

Zur 'l'echnik will ich nur bemerken, dass dis druckmessende Queckilbermanometer mit dem linken Aortenaste communicirte: die Bewegungen des Quceksibers wurden optisch rergrissert auf photographischem W'ege registrit. ba ich Gummimanometer nicht tagelang segen positiren Druck von $20.60 \mathrm{~mm} / \mathrm{Ig}$ dicht fand, musste ich aul dicse sonst ja cinwandsfreiere Duckmessung rer\%ichten. Dio There waren bloss zur Präparation mit Aether narliotisirt und nicht rurarisirt.

Das Resultat aller Versuche ist das, dass die Vergiftung mit Digitalisdosen, die gerade die Halbirung bringen, keinen negativen línfluss 
auf den Blutdruek äussert. Fin positive linfluss wurde oft im Ganzrhythmus nach der Vergiftung constatirt: über dic Genese dieser inconstanten Blutdrucksteigerung lässt sich nichts Bestimmtes aussagen.

Jch lasse Angaben über einige Blutdruckversuche folgen.

Versuche mit absteigenden Giftdosen.

14. Javuar 1905: 0,001 gigitalin lührt sehe raseh zur Halbirung und systolisehem Stillstand. Blutdruck sinkt continuirlich.

14. Januar 1905: 0,00075 g Digitalin

$\begin{array}{llllll} & & & & \text { Blutdruck: } & \text { Druckschwankungen } \\ \text { pro Systole: }\end{array}$

Resultat: Vorübergehende Blutdrucksteigerung unter Verminderung des Abfalls während der Diastole. Dic Halbirung unterbricht die Blutdrueksenkung nicht, der diastolische Abfall im Malbrhythmus ist sehr gross. Der verminderte Druckabfall von 5 Uhr 16 Min. bis 5 Uhr 18 Min. deutet auf Gefässwirkungr. Tödtliche Vergiftung.

14. Januar 05 Nachm.: 0,0005 Digitalin (töduliche Vergiftung)

Blutdruck: Diastol. Abfall:

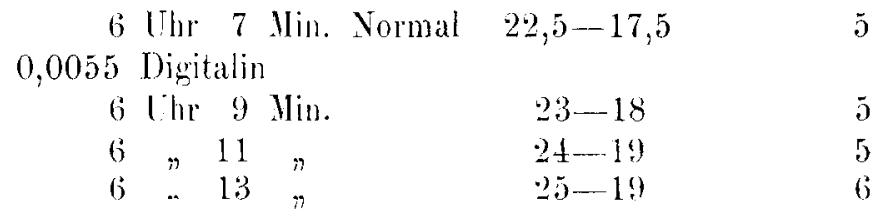

Resultat: Steigerung des Blutdrucks unter Constanz des diastolischen $\triangle$ bfalls vor Fintritt der llalbirung.

13. Januar 05: 0,00025 Digitalin (nicht tödtliche V'ergiftung) Blutdruck: Diastol. Abfall:

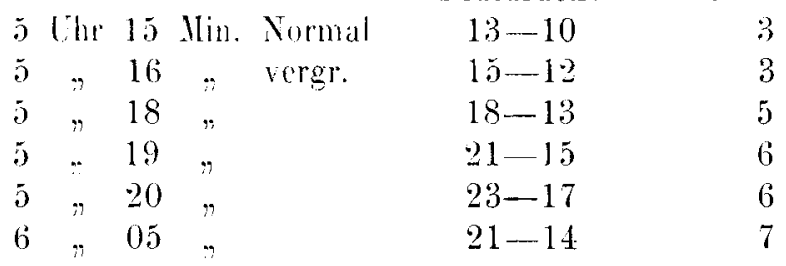

Resultat: Steigerung des Blutdrueks unter Zunahme des diastolischen Abfalls. Keine Halbirng während der zweistindigen Beobachtung. Dic Drueksteigerung geht vorüber.

1) A. h. im Momente der Systole des Ventrikels herrscht 35, der während der Diastole auf $30 \mathrm{~mm} \mathrm{llg}$ absinkt. Der Mitteldruck bleibt unberücksichtigt, da die Curren so eng geschrieben sind, dass der diastolische $\Lambda$ bfall zeitlich sich nicht bestinmen lässt. Im Halbrhythmus lient der Mitteldruck viel näher dem niedrigen Werthe der Drunlsebwankung. 
15. Januar 05: 0,00025 g Digitalin

Blutdruck: Diastol. Schwankung:
11 Uhr 45 Min. Normal
$41-36$
5

Verg. $0,00025 \mathrm{~g}$

$\begin{array}{llll}11 & \text { Uhr } & 47 & \text { Nin. } \\ 11 & n & 53 & n \\ 12 & n & 13 & n \\ 12 & " & 15 & n \\ 12 & n & 18 & "\end{array}$

$\begin{array}{ll}41-36 & 5 \\ 40-35 & 5 \\ 37-33 & 3 \\ 28-26 & 2\end{array}$

bis 12 Uhr 37 Min. Peristaltik und Habirung mit allmälig wachsenden Drucken und diastolischen $\Lambda$ blällen.

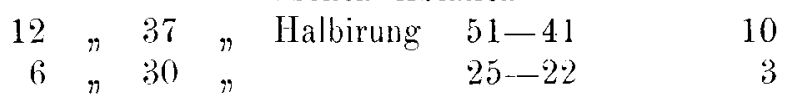

Resultat: Blutdrucksteigermng tritt erst nach der Halbirung auf unter Vergrösserung des diastolischen $\Lambda$ bfalls. Das Thier wurde weitere 36 Stunden unter ofterer Blutdruckregistrirung controlirt, es athmete während der ganzen Versuchsdaucr spontan. Das Hew arbeitete bis zum Ende der Beobachtung im halbirten Rhythmus. Der Druck schwankte während dieser Beobachtungsdauer zwischen 41 und 22. Bei Blutdruck $35 \mathrm{~mm}$ wurde der Versuch unterbrochen.

Auf Tafel XXI ist ein derartiger sehr lange danernder Blutdruckversuch mitgetheilt, zn dem noch folgende Erläuterungen zu geben sind.

Die Curven laufen ron linlis nach rechts, die Drucke waclisen ron oben nach unten; die oberste Linie ist Druck $=0$; Zeit $=5$ Sec. ('Tafel XXI).

1. Normalzustand. Frequenz 6 Pulse in 10 Sec, hei $x$ Injection ron $0,00025 \mathrm{~g}$ Digitalin in den Schenkellymphsack, zwischen 1 und 2 fehlen 2 Minaten.

2. Etwa das Stadium der rein positiven Wirkung am Ventriliel, bei $x$ bewegt sich das 'Thier. Frequenz dauernd 6 P'ulse in 10 Sec. Druck sinkt allmälig, die Druckschwankungen nehmen an Differenzgrösse ab, \%wischen 2 und 3 drei Min. Pause.

3. Freruenz ungeändert. Blutdruck sinlit weiter, zwischen 3 und 4 sechs Min. Pause.

4. Etwa Stadium der rein negativen Wirkung am Ventrikel (Füllungen wachsen; Drucke der isometrischen Zuckungen nehmen ab). Blutaruck sinkt weiter, Frequenz ungeändert, geringer diastolischer $A b f a l l(G e f a ̈ s s w i r l i n g ?$ ?), zwiscben 4 und 5 sechs Min. Panse.

5. Kritischer Beginn del Halbirung, am Ventrikel Teristaltik.

6. u. 7. Fortdauernde Peristaltik,

zwischen 7 und 8 zwei Min. Pause.

8. Regulärer Halbrhythmus. Frequent ; Pulse in 10 Sec. Blutdrucksteigerung, starker diastolischer Abfall.

9. Nach 3 Stunden. Halbrhythmus besteht noch, Iangsamer diastolischer Druckiablall (Gelässwirliung?).

10. 8 Stunden nach Beginn des V'ersuchs.

11. 24 Stunden nach Beginn des Versuchs. Frequenz hat zugenommen. 4 Pulse in 10 Sec.

12. 36 Stunden nach Beginn des Versuchs. 
13. 40 Stunden nach Beginn des Versuchs, von 12 ab weitere lreguenzunahme auf 5 Putse in 10 Sec. Vorsuch abyebrochen.

Die Erzwingung des Halbrlythmus durch Digitaliskörper ist für den Froseh jedenfalls ein ertragbarer Eingriff. In einer Bezichung lisst sich darum sogar ein günstiger Einfluss ableiten. Der arterielle Druckabfall im llalbrhythmus erreicht im Allgemeinen ${ }^{1}$ ) wesentlich tiefere Endwerthe als im Normalzustand, die Zuckung des Ventrikels wird demmach im HalbPhythms mit boherer Unterstiutzong erfolgen als in der Norm.

Persuche ron Gottlieb und llagnus am ausgeschnittenen Säugethierventrikel.

Die meisten Versuche einer wirklichen Analyse der Disitaliswirkung sind am Frosehherzen gemacht, dic rerallgemeinernden sehlüsse unter der Voraussetzung gezogen, dass in der Wirkung der Stoffo auf Herzen anderer thiere keine Wesensunterschiede rorhanden sind. Würden solehe Wesensuntersehicde vorhanden sein, so würden alle an Fröschen angestellten Untersuehungen nur einen an sich uninteressanten "Fall" beJeuchten, aber kcine allgemeine Bedeutung haben. Das wäre um so bedaucrlicher, als sich principielle Fragen doch am sichersten an dem technisch in weitesten Grenzen beherschbaren Erosch bearbeiten lassen.

Gottlieb und Ilagnus haben num eine Analyse der Digitaliswirkmn am isolirten Säugethicwentrikel ausgeführt und gefunden, dass bei constanter Anfangsspannung im Laufe der Digitalisvergiftung, und zwar während den Anfangsstadien derselben, die isotonisehen und isometrischen Zuckungen böhere Werthe erreiehen its vorher in Normalzistand, dabei ist die Frequenz nieht verlangsamt*).

Also das Pulsvolum (und damit auch dic Füllungen) gleicher Anfangsspannungen wächst im Laufe der Digitalisvergiftung; dies decki sich mit den Angaben von Frank und mir bezüglich des Froschborens (Sinken der Dehnungseurven der isotonischen Minima). Die Druckmaxima der isometrischen Zuckungen nehmen in G. u. M.' Versuchen gleichfalls zu; die zweite Erscheinung $\mathrm{kann}^{3}$ ) als Folge der ersten aufgefasst werden, wenn man annimmt, dass die Dehnungscurse der isotonischen Minima sinkt, bevor (zeitlich) die der isometrischen Maxima noch Veränderungen (siche oben, Seite 506) erleidet, dann herrscht bezüglich der isometrisehen Maxima das Gesetz des Normalzustandes (O. Erank), dass sie nämlich mit wachsenden effectiven Füllungen ${ }^{+}$) zunehmen. Da aber, wie gesagt,

1) In dem in Tafol XXI mitgetheilten Versuch ist dies nicht der lall.

2) Eine Dehnungscurve im frankschen Simne (also für alle Punkte der Relation: Anfangsspannung - Pulsfolum, resp. Pulsvolum-1)ruck) liann aus G. und M.'s Untersuchung nicht construit werden. Aus technischen Gründen bezw. Hindernissen mussten sich G. und A., um im Frank'schen Sinne zu sprechen, begnügen, die Wanderung eines Punltes der im übrigen unbekannten Dehnungscurve im zeitlichen Verlaufe der Digitalisvergiftung zu verfolgen.

3) Die Ergebnisse der G. u. M.'schen Versuche bei isometrischer und isotonischer Anordnung sind niclit unmittellar vergleichbar, da mit jedem lierzen jeweils nur sine Art der Thätigkieit untersucht werden konnte.

4) Aber nicht "Spannungen" was viplleicht anch fïr den Normalzustanu noch melur priacisirt werden musste. 
G. u. II.' Versuche keine construirharen Dehnungseurven liefern, lässt sich die Sache nicht entscheiden.

Es erscheint aber nach allem höchst wahrscheinlich, dass auch das Verhalten des Siiugethicruentrilicls unter Digitaliswirkung sich dem allgemeinen, für das Frosehher durchgelühten Prinzipe unterordnet.

\section{Der Halbrhythmus.}

Die von mir') gefundens Herabseczung der Frregloarkeit des Tentrikels nach Digitalistergiftung, ist eine lirscheinung für sich, die sich in ihren Folgen in den Gang der dynamischen Aeusserungen der Digitaliswirkung einschieben und hier mittelbar anscheinend neue Zustände schaffen kann. lch habe sehon in meiner ersten Vittheilung zu dem Thema die unmittelbaren Folgen der Habirung für den Kreislauf discutirt und war sehr geneigt, ju der Habirung das Wesen der "Heilwirkung" zu sehen. Da sich aber im Laufe dieser Untersuchung herausgestellt hat, dass sich schon mit Vergifungen geringeren Grades eine Wirkung auf den Kreislauf erziclen lässt, die im Simne der Strömungsgeschwindigkeit in den Arterien cine positive sein kann, rerliert diese meine Auffassung an Bedeutung.

Neuerdings betont Brandenburg ${ }^{2}$ ) diese Abnahme der Erregbarkeit stark, dagegen hat abor H. L. Hering's Sehüler Pletnew ${ }^{3}$ gezeigt, dass sie beim Warmblïter im Stadium des Ganzrhythmus nach der Digitalisvergiftung noch nicht nachzuweisen ist, cincm Stadium, wo nach Cottlieb und Magnus (l. c.) sowic meinen rorliegenden Beobachtungen, sehon eine dyamische Wirkung vorlanden ist. Ausserdem ist der Halbrhythmus zwar nicht für den Frosch icf. meine Blutdruckversuche), wohl aber für den Warmblüter (Pletnew u. a.) cin toxisches Vergiftungsstadium, also wohl schwer als Lnterlage der Heilwirkung zu gebrauchen.

Uebrigens scheint sich Brandenburg nicht darüber klar geworden zu sein, dass seine eigenen Untersuchungen sich auf das Stadium des Halbrhythmus bezichen, der nach seiner mehrfach geäusserten Ansicht eine "Absterbeerscheinung" sein soll. Meine Blutdruckversuche am Frosch haten ergeben, dass der Halbrhythmus nach kurzer Zeit sich cinstellt und dann Tage lang bestehen bleibt und sich registrieren lässt. Brandenburg zeigte, dass dassclbe zeitliche Verhältniss für die Erregbarkeitsabnahme besteht, wclche ihrerseits nach meinen früheren Darlegungen zum Halbrhythmus führen muss.

In lange damernden Versuchen braucht nun diese Frequenzverlangsamung nicht unbedingt und arithmetisch eine Halbirung zu sein.

Controllirt man die Pulszahl an gefensterten Fröschen, die zwischen der einzelnen Beobachtungen in ihren T'öpfen gehalten werden, nach einer Vergiftung, die am präparirten Thiere im Blutdruckversuch sicher die Halbirung bringt, so beobachtet man nicht selten, dass im Vergleioh zur

1) Arch. f. exp. Path. u. Pharmakiolog. Bd, 45, S. 346.

2) Zeitsehr. f. lilin. Med. 53. Band.

:3) Zeitschr. l. exper. Path. 1. Ther. 1904. 1. Ba. S. So. 
Normalfrequenz keine strenge llalbirung vorhanden ist, stets aber ist das Intervall zwischen den einzelnen Systolen beträchtlich vergrössert. Das Auftreten des Halbrhythmus ist num eine nothwendige Consequenz des Vergiftungsgrades, erseheint er nieht arithmetiseh genau, so wird der immerhin abnorme Zustand dureh regulatorische Innervation corrigirt sein, wozu gerade das Digitalinherz im Halbrhythmus besonders befähigt ist [Böhm¹, Brandenburg's]. So dürfte sich erklären, dass in Brandenburg's Versuchen nicht immer der Halbrhythmus sich constatiren lässt. lis schoint mir also abermals, dass die Harmonie zwischen meinen und Brandenburg's thatsïchlichen Ergebnissen eine erfreulich ausgedehnte ist.

Ich enthalte mich hier absichtlich jeder Speculation über den Mechanismus der Heilwirkung der Digitaliskörper, da die Hydrodynamik der Kroislaufanomalien, die dureh Digitalis corrigirt werden sollen, noch nicht innalysirt ist, wenn anch zugegeben werden muss, dass das Prineip dieser Ileilwirkung kein anderes sein kann, als die ITerbeifiilhung niner anch im Normalzustand möglichen Kireislaufainderung.

1) Pflüger's Archir. $1822 . \mathrm{Ba}$. $r$. S. 164.

2) Engelmann's Archir, 1904. Suppl. S. 213. 


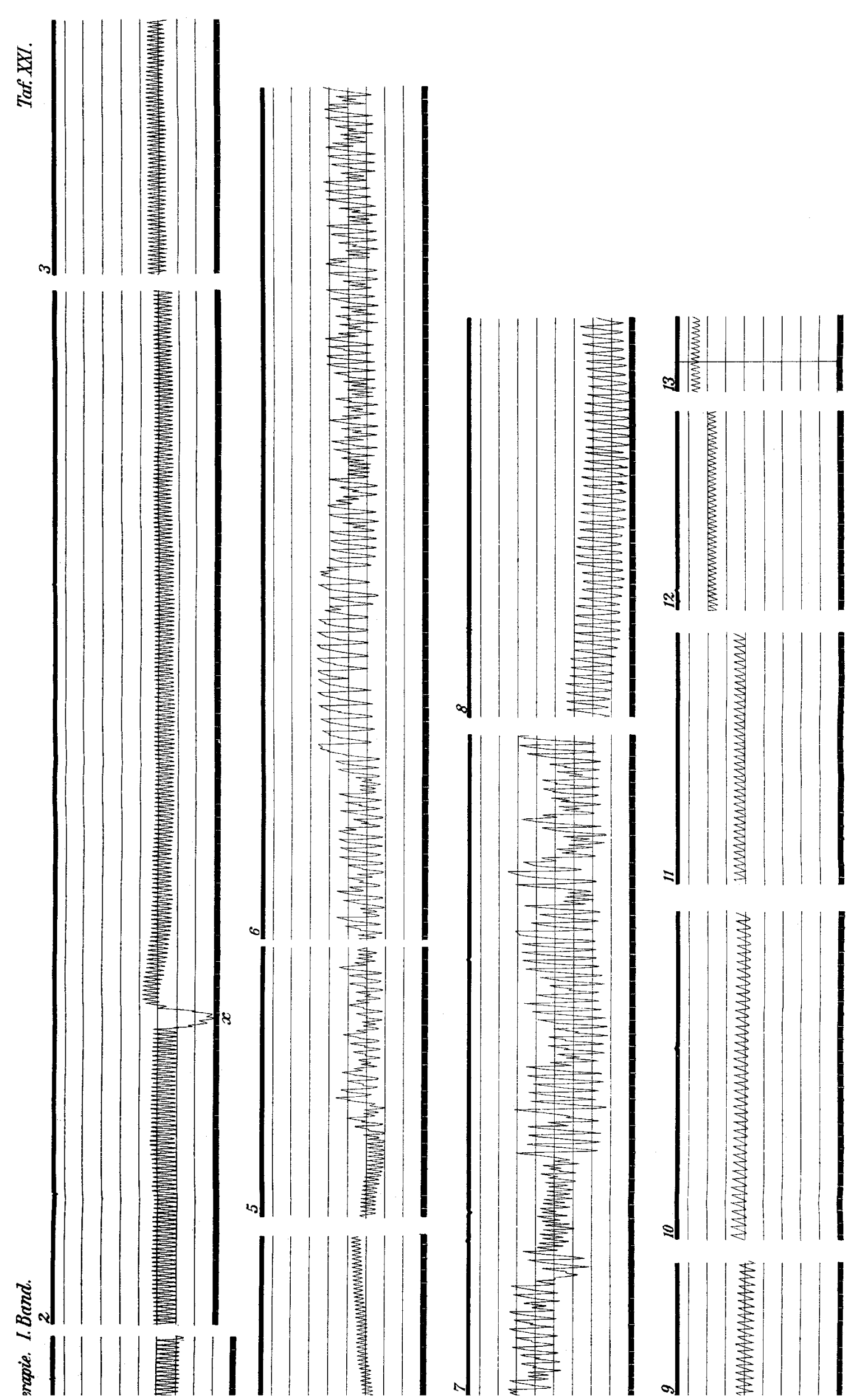

